\title{
Triggering of twists in solar prominence threads
}

\author{
Y. Taroyan ${ }^{1}$ and R. Soler ${ }^{2}$ \\ 1 Department of Physics, University of Aberystwyth, Aberystwyth SY23 3BZ, UK \\ e-mail: yot@aaber.ac.uk \\ 2 Departament de Fisica and Institut d'Aplicacions Computacionals de Codi Comunitari (IAC ${ }^{3}$ ), Universitat de les Illes Balears, \\ Palma de Mallorca, Spain \\ e-mail: roberto.soler@uib.es
}

Received 6 August 2019 / Accepted 3 October 2019

\begin{abstract}
Context. Magnetic twists are commonly associated with solar prominences. Twists are believed to play an important role in supporting the dense plasma against gravity as well as in prominence eruptions and coronal mass ejections, which may have a severe impact on the Earth and its near environment.

Aims. We used a simple model to mimic the formation of a prominence thread by plasma condensation with the aim of investigating the possibility of triggering twists during this process.

Methods. Temporal and spatial evolution of torsional Alfvénic perturbations driven by random photospheric motions was analysed using the linearised governing equations of motion and induction.

Results. We find that small amplitude perturbations are exponentially amplified in time as they propagate along the condensing thread. Mechanisms contributing to the rapid growth are explored. The result of the amplification process is the generation of large amplitude axisymmetric twists along the thread.

Conclusions. Magnetic twists may be triggered along a prominence thread when it is permeated by a converging flow, for example, during the evaporation and condensation of plasma along the thread. This may lead to the generation of vortices in the non-linear regime.
\end{abstract}

Key words. Sun: atmosphere - Sun: filaments, prominences - magnetohydrodynamics (MHD) - waves - instabilities

\section{Introduction}

Solar prominences are dense magnetic structures that are anchored in the visible surface known as the photosphere. They extend outwards into the Sun's upper atmosphere known as the corona. There are two key ingredients required for prominence formation: a magnetic structure, which may support the dense prominence plasma against gravity, and a mechanism to fill the structure with plasma. The formation process may take a few days, especially when the structure is located far from an active region (Parenti 2014).

A number of scenarios that fill the prominence structure with cool and dense plasma have been proposed (Gibson 2018). Some models invoke the direct injection of chromospheric plasma from a reconnection site into a pre-existing filament-channel field. Others suggest flux rope emergence from the photosphere and lifting into the corona by photospheric or chromospheric reconnection. In a third class of models, chromospheric material is evaporated into the corona and condenses to form the cool filament.

In the latter scenario, the formation of prominences has been explained in terms of thermal non-equilibrium between heating and radiative losses (Field 1965; Karpen 2015): heating at the feet of the magnetic field lines produces plasma evaporation and subsequent density accumulation at the location where the upflows meet. Density accumulation leads to enhanced radiation. If radiative losses dominate heating and if the structure is long enough, thermal collapse to low temperatures and the formation of a dense cool structure follows.
High-resolution observations show that solar prominences are formed by a myriad of thin and long sub-structures usually called threads, which are believed to outline particular lines of the prominence magnetic field (Martin 2015). The filling of the filament channel with plasma is not a homogeneous process. It can proceed by involving a pack of threads or thread segments, each of them occurring at different times (Luna et al. 2012).

Another key ingredient is the formation of a magnetic structure that may support the dense prominence plasma. If the curvature of a magnetic tube is concave towards the solar surface, it cannot support dense plasma against gravity. The existence of convex curvature in the magnetic field allows the retention of the condensed plasma and the formation of elongated cool structures. Models for dipped magnetic field lines range from sheared arcades to cylindrical flux ropes (see, for example, Terradas et al. 2015, 2016). A number of formation mechanisms have been proposed. Some of those are purely hypothetical and rely on basic arguments, whereas others, which are mainly based on magnetic reconnection, are backed up with highly sophisticated models. Examples include differential rotation or Coriolis forces in supergranules (Priest et al. 1989), reconnection between sheared field lines (van Ballegooijen \& Martens 1989), and the emergence of pre-twisted fields from beneath the photosphere (Rust \& Kumar 1994).

In a series of papers, Joarder and Roberts conducted analyses of the magnetohydrodynamic (MHD) modes of oscillation of a magnetised prominence slab embedded in the corona. They considered both longitudinal (Joarder \& Roberts 1992a) and perpendicular magnetic fields (Joarder \& Roberts 1992b). 
Dispersion relations were derived containing a variety of modes, which can be fast or slow, combined with kink or sausage as well as body or surface.

Prominence threads are dynamic and rapidly changing structures (Engvold 2015) and often display transverse oscillations with periods of several minutes (Lin 2011). Thread transverse oscillations have been theoretically interpreted as transverse MHD waves (Terradas et al. 2008). The analysis of these oscillations can provide indirect information about the physical and geometrical properties of the threads (Soler et al. 2010, 2015).

Effects of mass flows and non-adiabatic processes on the oscillations of an individual prominence thread have also been modelled (Soler et al. 2008). A review of prominence oscillations and prominence seismology can be found in Arregui et al. (2018).

Observations of solar tornadoes made by Pike \& Mason (1998) suggested that rotation may play an important role in the dynamics of the solar transition region. More recently, solar tornadoes were observed in detail with the Atmospheric Imaging Assembly (AIA) onboard the Solar Dynamics Observatory (Lemen 2012). Li et al. (2012) detected plasma moving along spiral paths with an apparent rotation that lasted for more than three hours. Wedemeyer-Böhm et al. (2012) showed that magnetic tornadoes are associated with vortex flows in the photosphere. These events are observed with the Swedish $1 \mathrm{~m}$ Solar Telescope (Scharmer et al. 2003) as "chromospheric swirls". Wedemeyer et al. (2013) suggested that that the majority of solar tornadoes are the legs of prominences and filaments. The tornado structure may rotate as a whole but only the thin threads, which compose the tornado base, trace the rotation. The rotation of the tornadoes may progressively twist the magnetic structure of the prominence until it becomes unstable and erupts.

In the context of solar prominences, MHD waves are primarily studied for diagnostic purposes. The present paper considers torsional Alfvénic perturbations in conjunction with time-dependent converging flows due to accretion of mass, for example, during the formation stage of a prominence. We propose a wave based mechanism to trigger the observed vortices and the twists required for prominence support.

In general, convection in the photosphere is expected to excite both vertical and horizontal motions. Carlsson \& Stein (1997) used the observed vertical velocity as a driver of acoustic waves in their numerical simulations. Hillier et al. (2013) suggested that transverse oscillations of prominence threads might be driven by horizontal motions in the photosphere, where the field lines are anchored. On the other hand, Matsumoto \& Shibata (2010) used an observed temporal spectrum of horizontal granular motions in the photosphere to mimic generation of torsional Alfvén waves.

The present paper investigates the evolution of torsional Alfvén waves or twists in the linear regime. An important property of linear twists is that they are decoupled from other types of perturbations. Therefore, twists can be considered without the need to address the evolution of other perturbations driven by the photosphere. However, coupling is expected to occur in the presence of transverse inhomogeneities or in the non-linear regime, when the amplitudes become sufficiently large.

The mechanism of twist amplification has been studied in the linear regime in the context of upflows (Taroyan 2015) and intergranular downdrafts (Taroyan \& Williams 2016). The back reaction of the amplified twists on the mass flows and their non-linear evolution has been studied by Williams et al. (2016), Williams \& Taroyan (2018).

The paper is constructed as follows: the prominence thread model and the governing equations are presented in Sect. 2;
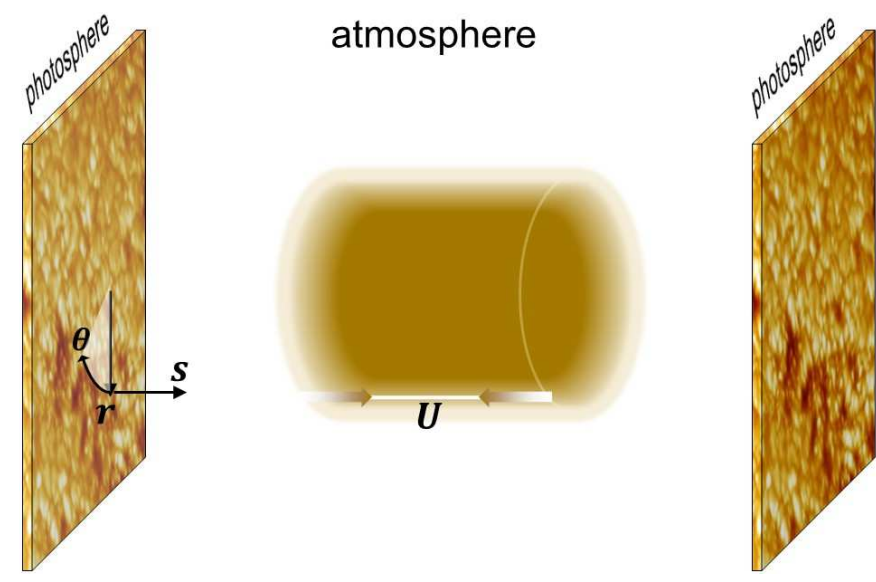

Fig. 1. Axially symmetric structure is embedded in corona and rooted in photosphere at both ends. A single magnetic field line representing a thin prominence thread is permeated by inflow $U$ in the longitudinal $s$-direction. Small-amplitude axisymmetric twists $(\partial / \partial \theta)$ are driven at both footpoints by photospheric motions. The thread is at a distance $r$ from the axis of symmetry.

we derive the boundary conditions at the footpoints in Sect. 3; Sect. 4 presents analysis of twist evolution; the main conclusions of the work are summarised in Sect. 5.

\section{Model and governing equations}

\subsection{Mass loading along a prominence thread}

We represent a thin prominence thread by a single magnetic field line of length $L$. The distance measured along it is denoted by $s$ (Fig. 1). The magnetic field strength, $B_{0}$, is assumed to be constant for simplicity. The effect of gravity is not taken into account. In order to mimic the process of mass loading in the thread due to evaporation and condensation, we consider a plasma density which increases around the centre of the thread as time goes on.

Empirical models such as VAL (Vernazza et al. 1981) provide density as a prescribed function of height in the low atmosphere. Due to lack of detailed information on the variation of density, magnetic field, and flows in the lower atmosphere during prominence formation we reduce the photosphere/chromosphere environment to a boundary which is perturbed by convective motions. The density at the footpoints is kept fixed for all times, assuming an infinite reservoir of mass exists.

After Soler et al. (2015), we prescribe the longitudinal dependence of density using a Lorentzian function, namely

$\rho(s, t)=\frac{\chi(t) \rho_{0}}{1+4(\chi(t)-1) s^{2} / L^{2}}$,

where $s$ denotes the direction along the magnetic field, with $s=0$ and $s= \pm L / 2$ corresponding to the thread centre and feet, respectively; $\rho_{0}$ is the uniform density at $t=0$. The time-dependent ratio of the central density to the footpoint density,

$\chi(t)=1+\left(\frac{t}{\tau}\right)^{2}$,

where $\tau$ is a constant characterising the rate of mass accumulation.

For the prescribed Lorentzian profille (1), the longitudinally averaged density varies with time as $\langle\rho\rangle \approx \rho_{0} \pi t /(2 \tau)$ when $\chi(t) \gg 1$. The linear growth in this expression is consistent with 



Fig. 2. Density (left panel) and flow velocity (right panel) are displayed as functions of time $\left(t / t_{\mathrm{A}}\right)$ and distance along the thread $(s / L)$. Each plot combines an image (bottom), a surface plot (middle), and a contour plot (top) of the corresponding quantity. Density is normalised with respect to its initial value, $\rho_{0}$. The velocity of the flow is normalised with respect to initial Alfvén speed, $c_{\mathrm{A} 0}$.

Luna et al. (2012), where the mass of the condensations grows linearly. For the rest of the paper a growth rate of $\tau=8 t_{\mathrm{A}}$ is selected, which is similar to the linear growth rate adopted by Luna et al. (2012). The quantity $t_{\mathrm{A}}$ is the Alfvénic travel time and is defined later.

Mass loading in the thread is physically caused by the presence of a field-aligned compressible flow. The flow velocity, $U$, that is consistent with the prescribed density, $\rho$, satisfies the continuity equation, namely

$\frac{\partial \rho}{\partial t}+\frac{\partial}{\partial s}(\rho U)=0$.

The continuity Eq. (3) shows that an increasing density $(\partial \rho / \partial t>$ 0 ) implies a converging flow - an inflow through both footpoints.

The present study assumes a constant magnetic field, $B_{0}$, along the thread. A variable field will affect the flow profile through an extra term in the continuity Eq. (3). A stronger magnetic field in an added photosphere/chromosphere is likely to have a positive effect on the flow speed and its gradient, whereas higher density is likely to have the opposite effect.

The gas pressure that is consistent with the present prescription of density and flow velocity can be determined from the longitudinal component of the momentum equation which may also contain extra force terms. However, gas pressure has no effect on the Alfvénic perturbations and we can therefore afford to ignore it in our analysis as long as the amplitudes of the perturbations remain small. The longitudinal component of the momentum equation should be included in the analysis during the non-linear stage of evolution.

We normalise distance with respect to the thread length, $L$. Density and Alfvén speed are normalised with respect to the initial uniform density, $\rho_{0}$, and Alfvén speed, $c_{\mathrm{A} 0}=B_{0} / \sqrt{\mu_{0} \rho_{0}}$, where $\mu_{0}$ is the permeability of free space. Therefore, time is normalised with respect to the Alfvénic travel time $t_{\mathrm{A}}=L / c_{\mathrm{A} 0}$.

Figure 2 displays the evolution of density and mass flow along a thread for $0<t<50 t_{\mathrm{A}}$. The thread is permeated by a converging flow of plasma $U$ along the magnetic field which leads to accumulation of mass. The flow reaches a nearly stationary state in about $10 t_{\mathrm{A}}$. There is change of sign at the stagnation point at $s=0$. The highest speeds of around $0.1 c_{\mathrm{A} 0}$ that are reached near the footpoints are much lower than the corresponding Alfvén speeds. The final centre-to-footpoint density ratio is $\chi_{\max } \approx 40$. Both the density and the flow profiles are symmetric with respect to the origin at $s=0$. This background configuration is used in all the following simulations.

\subsection{Torsional perturbations}

Let the thread shown in Fig. 1 be at a distance $r \neq 0$ from the axis of symmetry of the structure representing a solar prominence, where the magnetic field, $B_{0}$, is in the axial direction, $s$. We introduce an azimuthal angle, $\theta$, measured about the prominence symmetry axis and consider only axisymmetric motions, so $\partial / \partial \theta=0$. Small amplitude torsional Alfvénic perturbations are governed by the azimuthal $(\theta)$ components of the linearised equations of momentum and induction:

$$
\begin{aligned}
& \frac{\partial v_{\theta}}{\partial t}+U \frac{\partial v_{\theta}}{\partial s}=\frac{B_{0}}{\mu_{0} \rho} \frac{\partial b_{\theta}}{\partial s}, \\
& \frac{\partial b_{\theta}}{\partial t}+U \frac{\partial b_{\theta}}{\partial s}+b_{\theta} \frac{\partial U}{\partial s}=B_{0} \frac{\partial v_{\theta}}{\partial s},
\end{aligned}
$$

where $v_{\theta}$ and $b_{\theta}$ are the azimuthal velocity and magnetic field perturbations. We note that gas pressure plays no role in the evolution of Alfvén perturbations. Since linear perturbations are considered, the back reaction of the perturbations on the background plasma flow is neglected.

Equations (4) and (5) can be combined to derive an energy equation (Taroyan \& Williams 2016):

$\frac{\partial W_{\mathrm{T}}}{\partial t}+\frac{\partial F_{\mathrm{W}}}{\partial s}=-\frac{\partial U}{\partial s} W_{\mathrm{m}}$ 
where $W_{\mathrm{T}}$ is the sum of the kinetic and magnetic energy densities:

$W_{\mathrm{T}}=W_{\mathrm{k}}+W_{\mathrm{m}}=\frac{\rho v_{\theta}^{2}}{2}+\frac{b_{\theta}^{2}}{2 \mu_{0}}$

and

$F_{\mathrm{W}}=U W_{\mathrm{T}}-\frac{B_{0}}{\mu_{0}} v_{\theta} b_{\theta}$

is the energy flux. The source term on the right-hand side of the energy equation represents the coupling between the twists and an accelerating/decelerating flow that occurs along the entire length of the thread. We note that the energy Eq. (6) remains valid in the non-linear regime (Williams \& Taroyan 2018).

The analysis of twist amplification cannot be reduced to standard stability analysis which involves setting the linear perturbations proportional to $\exp (-i \omega t)$ and solving the resulting eigenvalue problem for the complex frequency $\omega$. This procedure fails because the mass condensation implies a timedependent background. Therefore the coefficients in the governing Eqs. (12) and (13) depend both on time and distance. This means that normal mode analysis is no longer applicable.

One could instead set a long condensation time-scale compared with the time-scale of the perturbations and tackle the problem via the WKB approximation - an approach adopted by, for example, Erdélyi et al. (2011) who studied the damping of longitudinal magneto-acoustic oscillations in slowly varying coronal plasma. See also Ballester et al. (2018). A similar approach could be adopted when the ratio $U / c_{\mathrm{A}}$ is small. Otherwise a different approach, fully numerical, is required.

\section{Boundary conditions}

Taroyan \& Williams (2016) considered twist amplification as an initial value problem in a stratified infinite domain. A timeindependent unidirectional downflow was present. They showed that magnetic twists are amplified even when fluxes through boundaries are eliminated by imposing boundary conditions with vanishing perturbations at $\pm \infty$.

Eliminating fluxes through the boundaries, namely, the energy flux and the azimuthal magnetic flux, allows one to minimise the role of boundaries and study the pure effect of flowtwist coupling.

The present paper considers a thread of finite length $L$. Fluxes can be eliminated by imposing vanishing boundary conditions, $\left.v_{\theta}\right|_{s= \pm L / 2}=0,\left.b_{\theta}\right|_{s= \pm L / 2}=0$. However, it is easy to check that these conditions are incompatible with the governing Eqs. (4) and (5) unless the flow speed matches the Alfvén speed at the boundaries. Figure 2 shows that for the parameter values chosen for this study, the ratio of the flow speed to the Alfvén speed remains less than 0.1 at the boundaries throughout the process of condensation. Therefore, a different approach needs to be adopted.

We consider twists propagating along the magnetic field that are driven by photospheric motions. The photospheric boundaries are located at $s= \pm L / 2$. For a static atmosphere with $U=0$ this is consistent with the following boundary conditions:

$v_{\theta}=f^{ \pm}(t)$, at $s= \pm \frac{L}{2}$,

where $f^{ \pm}(t)$ are the photospheric drivers at the two footpoints. The second condition naturally follows from Eq. (4):

$\frac{\partial b_{\theta}}{\partial s}=\frac{\mu_{0} \rho_{0}}{B_{0}} \frac{\partial f^{ \pm}}{\partial t}$, at $s= \pm \frac{L}{2}$,

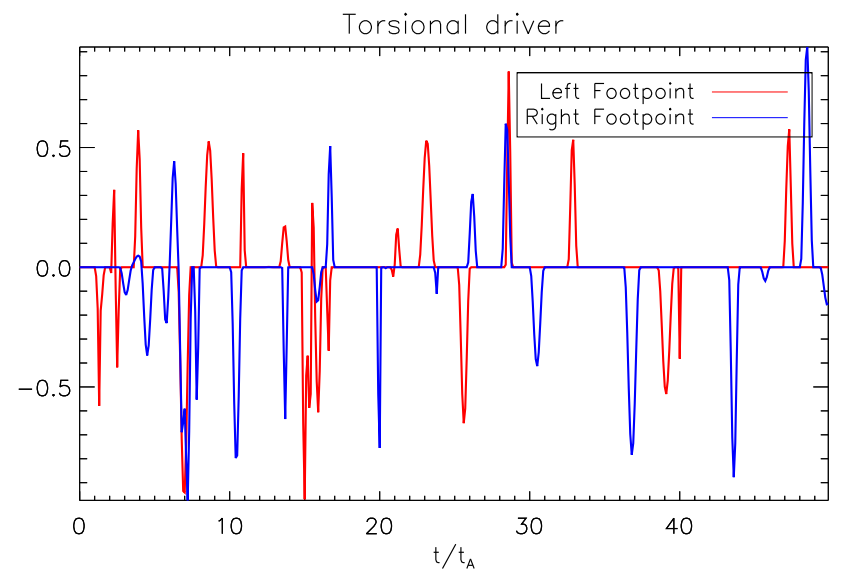

Fig. 3. Photospheric driver of torsional Alfvénic perturbations is modelled as a superposition of random smooth pulses. The red curve represents the driver at the left footpoint, $f^{-}$, and the blue curve represents the driver at the right footpoint, $f^{+}$. Each pulse has a random duration between $0.1 t_{\mathrm{A}}$ and $t_{\mathrm{A}}$, offset time, and amplitude. The amplitudes are measured in arbitrary units with a maximum of 1 .

where $\rho_{0}$ is the density at $s= \pm L / 2$. However, with the introduction of flow the boundary conditions (9) and (10) are no longer compatible with Eq. (4). Indeed, for Eq. (4) to be consistent with the boundary conditions (9), (10), an additional third condition, $\partial v_{\theta} / \partial s=0$, would be required.

In order to impose consistent boundary conditions we introduce a new variable,

$w_{\theta}=U \rho v_{\theta}-\frac{B_{0} b_{\theta}}{\mu_{0}}$,

and rewrite Eqs. (4) and (5) in the following form:

$$
\begin{aligned}
& \frac{\partial}{\partial t}\left(\rho v_{\theta}\right)=\frac{\partial w_{\theta}}{\partial s}, \\
& \frac{\partial w_{\theta}}{\partial t}+2 U \frac{\partial w_{\theta}}{\partial s}+\frac{\partial U}{\partial s} w_{\theta}+\left(U \frac{\partial \rho}{\partial t}-\rho U \frac{\partial U}{\partial s}-\rho \frac{\partial U}{\partial t}\right) v_{\theta} \\
& +\rho\left(c_{\mathrm{A}}^{2}-U^{2}\right) \frac{\partial v_{\theta}}{\partial s}=0 .
\end{aligned}
$$

We still assume that the magnetic field lines are perturbed at the photospheric level so that $v_{\theta}=f^{ \pm}(t)$ at $s= \pm L / 2$. The second set of boundary conditions naturally follows from Eq. (12). In summary, the new boundary conditions are:

$$
\begin{aligned}
& \left.v_{\theta}\right|_{s= \pm \frac{L}{2}}=f^{ \pm}(t), \\
& \left.\frac{\partial w_{\theta}}{\partial s}\right|_{s= \pm \frac{L}{2}}=\frac{\partial}{\partial t}\left(\rho_{0} f^{ \pm}\right) .
\end{aligned}
$$

Conditions (14) are known as essential, whereas conditions (15) are known as natural boundary conditions. The new set of governing Eqs. (12) and (13) combined with the boundary conditions (14) and (15) represents a well-posed problem which is tackled numerically.

Solutions are obtained in terms of $v_{\theta}$ and $w_{\theta}$. The variable $b_{\theta}$ can be expressed in terms of these solutions using Eq. (11). Test runs show that for small ratios, $\left|U / c_{\mathrm{A}}\right|_{s= \pm L / 2}$, solutions obtained with the boundary conditions (14) and (15) are similar to those obtained with the boundary conditions (9) and (10). However, differences become more and more pronounced when the ratio increases.

The functions $f^{ \pm}(t)$ used here are periodic or random pulses at the photospheric boundaries. In the first case the boundaries 

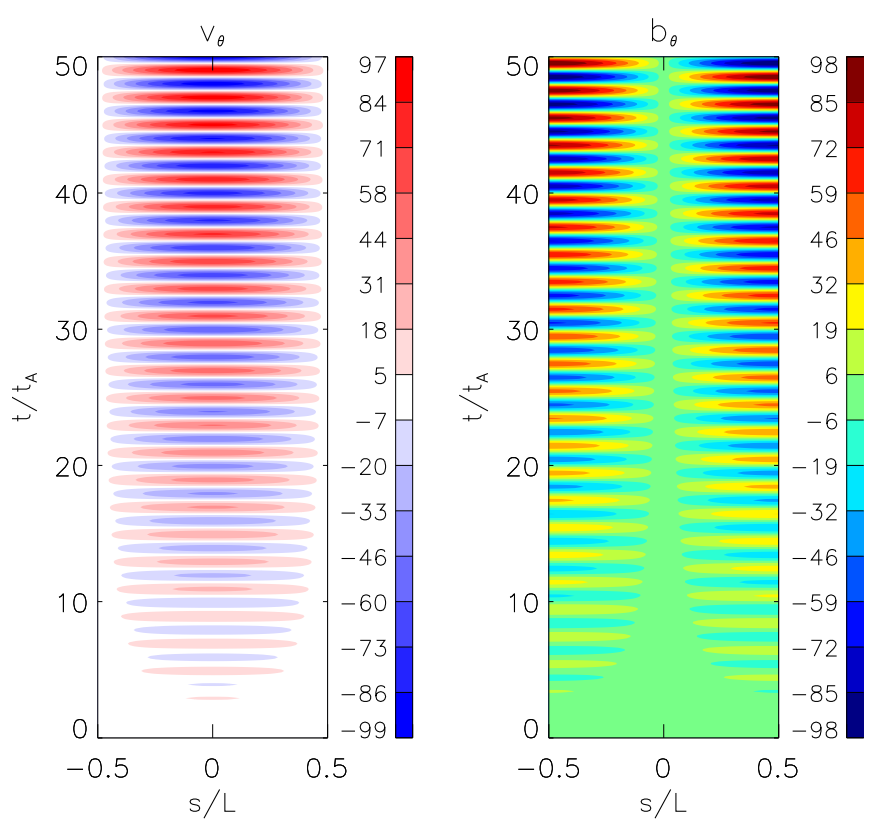

Fig. 4. Case I: spatial-temporal evolution of $v_{\theta}$ (left panel) and $b_{\theta}$ (right panel) in a static background. Colour bars display the range of values in each panel. The perturbations are measured in arbitrary units. A periodic driver with an amplitude of 1 is applied at the footpoints at $s=-L / 2$ and $s=-L / 2$.

are driven at the fundamental mode period $2 t_{\mathrm{A}}$ using the following expression for the functions $f^{ \pm}(t)$ :

$f^{ \pm}(t)=\sin \left(\pi \frac{t}{t_{\mathrm{A}}}\right)$

In the second case, they are constructed as a superposition of smooth random pulses of the form

$f^{ \pm}(t)=\sum_{i=0}^{n} f_{i}^{ \pm}(t)$

where

$f_{i}^{ \pm}(t)= \begin{cases}A_{i}^{ \pm} \sin ^{2}\left(\pi \frac{t-t_{i}^{ \pm}}{\tau_{i}^{ \pm}}\right), & \text {if } t_{i}^{ \pm}<t<t_{i}^{ \pm}+\tau_{i}^{ \pm}, \\ 0, & \text { otherwise. }\end{cases}$

Each pulse, $f_{i}^{ \pm}$, has a randomly assigned velocity amplitude, $A_{i}^{ \pm}$. Since wave amplitude is irrelevant for linear waves, we use a random number generator that assigns values between -1 and 1 in arbitrary units. Each pulse is offset at random time, $t_{i}^{ \pm}$, and has random duration, $\tau_{i}^{ \pm}$, where $0.1 t_{\mathrm{A}}<\tau_{i}^{ \pm}<t_{\mathrm{A}}$. A total of $n=25$ pulses are launched from each footpoint.

An outcome of the above described procedure is shown in Fig. 3. The blue and red curves represent the random photospheric driver profiles at the right and left footpoints. It can be seen that some pulses overlap. The purpose of applying a random velocity driver is to eliminate any resonance effects and to show that any wave amplification is independent of the form of the imposed driver profile.

\section{Analysis of twist evolution}

We integrate the set of linear partial differential Eqs. (12) and (13) numerically using a general-purpose solver (PDE_ MOL) from the Advanced Maths and Statistics Library for IDL.
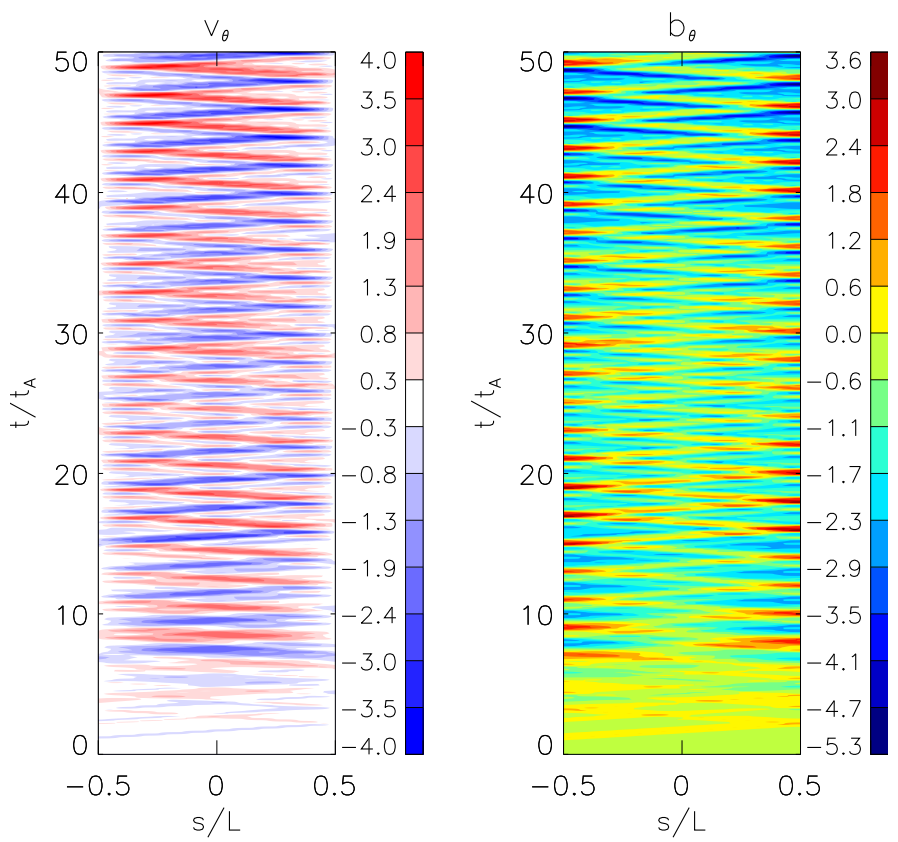

Fig. 5. Case II: spatial-temporal evolution of $v_{\theta}$ (left panel) and $b_{\theta}$ (right panel) in a static background. The perturbations are measured in arbitrary units. A random driver shown in Fig. 3 is applied at the footpoints.

It is based on the method of lines that proceeds by discretising the spatial derivatives and leaving the time variable continuous (Hamdi et al. 2007). We use a uniform spatial grid of 1000 points.

The structure is initially at rest with no background flow and no perturbations. The linear perturbations and the driver amplitudes at the boundaries are expressed in arbitrary units. We conduct four experiments (Cases I-IV) with the same duration of $50 t_{\mathrm{A}}$.

\subsection{Case I: static background, periodic driver}

Before investigating the effects of flow it is instructive to consider the temporal evolution of perturbations in a static system. Two different types of drivers are selected. We first consider the case when the two boundaries at $s= \pm L / 2$ are driven periodically. A resonant period matching a normal mode oscillation period of the system is selected. Amplitudes are expressed in arbitrary units and we choose a driver amplitude of one.

Figure 4 displays the spatio-temporal evolution of the perturbations. The resonant driver sets up a standing wave with a period of $2 t_{\mathrm{A}}$. The amplitude of this fundamental mode standing wave grows in time by a factor of about 100 in $50 t_{\mathrm{A}}$. The standing wave pattern is apparent both in $v_{\theta}$ and $b_{\theta}$. The magnetic field perturbation has a node at $s=0$, where the velocity perturbation has an antinode.

We conclude that twist amplification may occur in the absence of an inflow if the driver period matches a natural oscillation period of the system. The periodic driver at the boundaries continuously pumps energy into the system. This injected energy amplifies in time the excited standing wave.

\subsection{Case II: static background, random driver}

However, the photospheric boundary is more likely to act as a random driver due to the random nature of convective motions. 

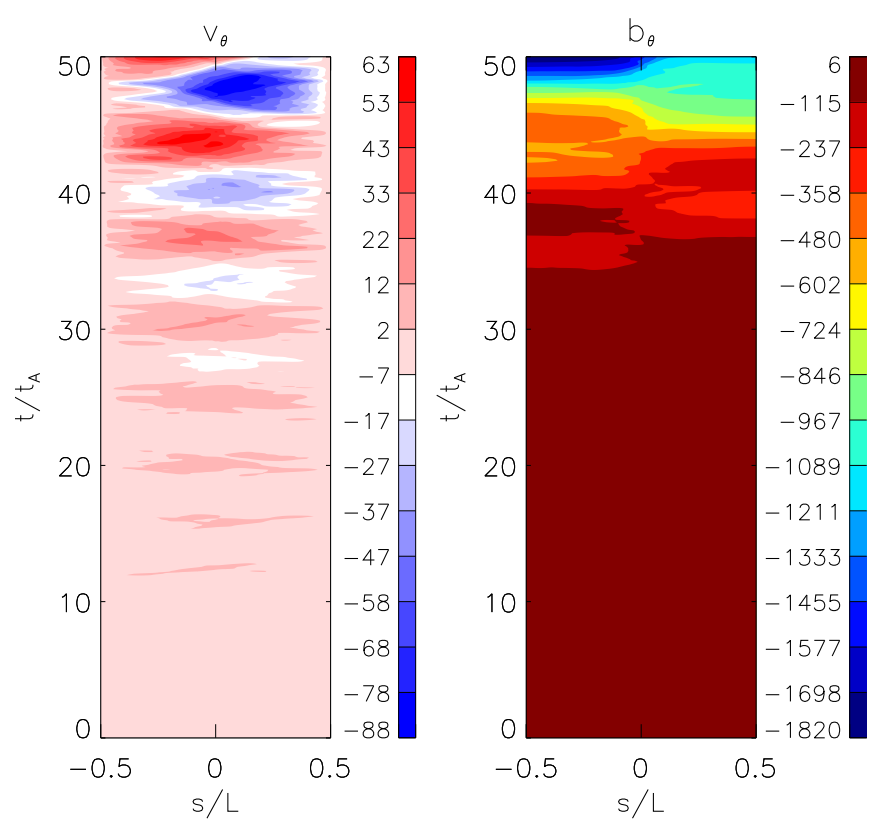

Fig. 6. Case III: spatial-temporal evolution of $v_{\theta}$ (left panel) and $b_{\theta}$ (right panel) during mass condensation. The perturbations are measured in arbitrary units. A random driver shown in Fig. 3 is applied at the footpoints.

We choose the velocity perturbations, $f^{ \pm}(t)$, to represent smallscale Alfvénic disturbances that are shown in Fig. 3. Here we do not care about the nature of this Alfvénic noise.

Figure 5 shows the temporal evolution of $v_{\theta}$ and $b_{\theta}$ along the field. The background is still assumed to be static. After the waves are set up no significant amplification can be detected. Unlike Case I, the driver is not resonant with a normal mode of the thread. There are no sources of amplification and, therefore, the obtained result is expected. Both $v_{\theta}$ and $b_{\theta}$ exhibit propagation along the thread in Fig. 5. This propagation is represented by narrow stripes slightly inclined from the horizontal direction. The propagation speed in both directions is determined by the Alfvén speed, $c_{\mathrm{A}}$.

\subsection{Case III: mass condensation, random driver}

The inclusion of flow has a dramatic effect on the temporal evolution of the torsional motions. We apply the same boundary conditions shown in Fig. 3. Unlike Case II, where the amplitudes remain small, there is significant amplification both in $v_{\theta}$ and $b_{\theta}$.

Similar to the previous case, propagation along the thread in opposite directions can be identified in the left panel of Fig. 6 in the form of non-horizontal red and white/blue stripes. The propagation speed in both directions is still mainly determined by the Alfvén speed, $c_{\mathrm{A}}$, whereas the flow has a minor role due to lower speeds. However, the Alfvén speed is now variable both in time and along the thread. It decreases due to accumulation of plasma towards the centre of the thread. A direct consequence of a decreasing propagation speed is the broadening of red and blue features identified in the left panel of Fig. 6 as time goes on.

There is significant increase in $b_{\theta}$ compared with $v_{\theta}$. Towards the end of the simulation the values in the right panel of Fig. 6 are about three orders of magnitude higher than the starting values. It is also interesting to note that unlike $v_{\theta}$, the magnetic field perturbation, $b_{\theta}$, does not change sign towards the end of the simulation as it becomes increasingly negative. This behaviour is explained later in terms of magnetic flux variation.
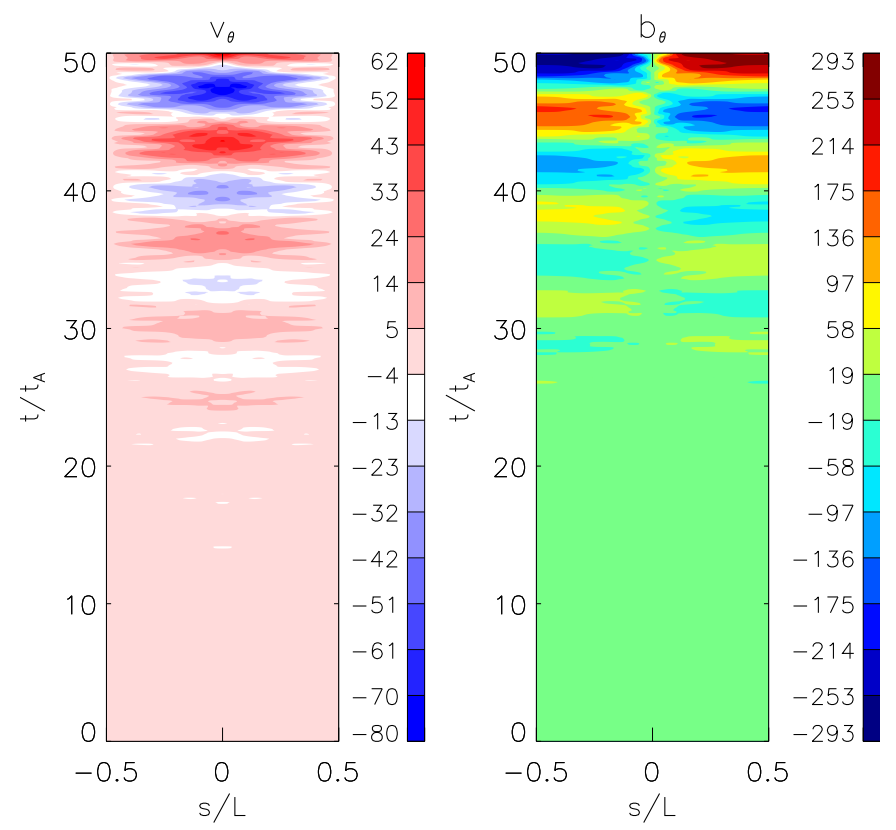

Fig. 7. Case IV: spatial-temporal evolution of $v_{\theta}$ (left panel) and $b_{\theta}$ (right panel) during mass condensation. The perturbations are measured in arbitrary units. A random driver represented by the red curve in Fig. 3 is applied at the footpoints.

We conclude that a combination of plasma flow and small torsional Alfvénic perturbations results in amplification of the latter which may lead to twisting of magnetic field lines and triggering of vortex motions about the axis of symmetry if the system is left to evolve into the non-linear regime.

\subsection{Case IV: mass condensation, random symmetric driver}

Finally, we consider the case when the footpoint drivers are symmetrically random: the same driver represented by the red curve in Fig. 3 is applied at both footpoints. The purpose of including this case in the analysis will become clear in the next subsection.

The results are shown in Fig. 7. There are similarities with Case III but there are also differences. By the end of the experiment the amplitudes increase more compared with the resonant driver in Case I. However, the magnetic field perturbation, $b_{\theta}$, is almost an order of magnitude lower compared with the corresponding values in the case of an asymmetrically random driver (Case III)

It is also interesting to note that $b_{\theta}$ continues to change sign until the end of the experiment unlike in Case III. The change occurs at the centre where a current sheet is gradually forming. The role of this current sheet in the subsequent evolution of the prominence including possible eruption can only be investigated using a multi-dimensional non-linear model. That is beyond the scope of the present work.

The symmetry in the driver profile is also responsible for the quasi-standing wave patterns seen in both panels of Fig. 7, where the wave period increases in time due to decreasing Alfvén speed. However, it is important to emphasise that the driver is random with no preferred periodicity.

In summary, we have obtained amplification in two possible scenarios, namely when the photospheric driver is resonant (Cases I) or when a converging flow towards the centre is present (Cases III and IV). We conclude that the effect of flow on the amplification of torsional Alfvénic perturbations is more pronounced compared with the effect of a resonant photospheric 

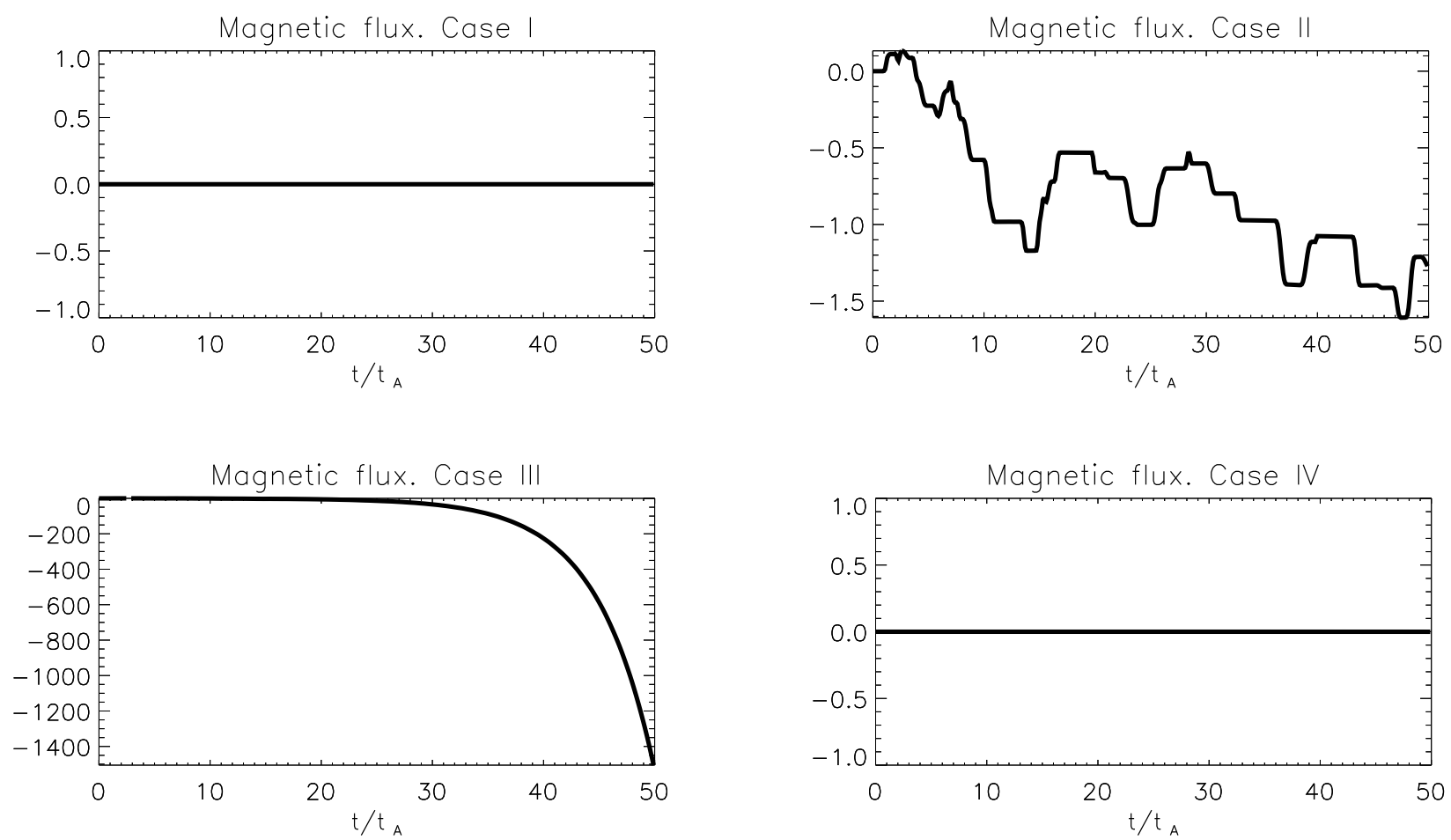

Fig. 8. Azimuthal magnetic flux along the thread is shown as a function of normalised time for Cases I-IV.

driver. In the former case, amplitude growth occurs even when the footpoints are driven randomly. The source of amplification is therefore associated with the flow. We next discuss potential causes behind the amplification process.

\subsection{Magnetic flux evolution}

An inflow through the footpoints could lead to convergence of azimuthal magnetic flux into the thread. Magnetic flux convergence is therefore a potential driver of twist amplification.

The total azimuthal magnetic flux along the thread is defined as $\int_{-L / 2}^{L / 2} b_{\theta} \mathrm{d} s$. From the induction Eq. (5) we have:

$$
\int_{-L / 2}^{L / 2} b_{\theta} \mathrm{d} s=-\int_{0}^{t}\left[F_{\mathrm{M}}(L / 2)-F_{\mathrm{M}}(-L / 2)\right] \mathrm{d} t,
$$

where

$F_{\mathrm{M}}=U b_{\theta}-B_{0} v_{\theta}$.

Equations (19) and (20) show that, in general, the total flux is not conserved.

We consider the evolution of total azimuthal magnetic flux in Fig. 8. The flux remains zero in the case of a periodic driver (Case I) despite an increase in the amplitudes. A similar picture can be seen for Case IV, where the inflow is combined with a random symmetric driver. Therefore twist amplification may occur even when the total magnetic flux remains zero regardless of the presence or absence of flow. A moderate variation can be seen for Case II, where the random driver is asymmetric and the flow is absent. There is strong convergence of magnetic flux for Case III, which is comparable with the significant amplitude increase in the right panel of Fig. 6 .

The right panel of Fig. 6 shows no change of sign for $b_{\theta}$ towards the end of the experiment. It becomes increasingly negative along the thread as time goes on. This behaviour in Case III can be explained in terms of magnetic flux convergence which leads to increasingly negative flux in the lower left panel of Fig. 8. The increase may be in the positive direction when a different random driver is applied at the photospheric level.

We conclude that magnetic flux convergence contributes to amplitude growth. However, it does not represent the main source of amplification which may occur even when the total flux remains equal to zero.

Other potential contributors to the amplification process include the influx of azimuthal energy through the footpoints and the mechanism of twist-flow coupling along the thread. Both are considered in the next subsection.

\subsection{Energy evolution}

In order to understand the mechanism of amplification during the mass condensation, we derive an equation of integrated azimuthal energy from Eq. (6):

$\frac{\partial E_{\mathrm{T}}}{\partial t}+F_{\mathrm{W}}\left(\frac{L}{2}\right)-F_{\mathrm{W}}\left(-\frac{L}{2}\right)=-\int_{-L / 2}^{L / 2} \frac{\partial U}{\partial s} W_{\mathrm{m}} \mathrm{d} s$,

where $E_{\mathrm{T}}$ is the total energy of the perturbations along the thread, $F_{\mathrm{W}}$ is the energy flux defined by Eq. (8), and the integral on the right-hand side represents a source term. A positive flux at the left footpoint, $s=-L / 2$, and a negative flux at the right footpoint, $s=L / 2$, contribute to energy increase. The timeintegrated net influx of azimuthal energy,

$I_{\mathrm{E}}=-\int_{0}^{t}\left[F_{\mathrm{W}}\left(\frac{L}{2}\right)-F_{\mathrm{W}}\left(-\frac{L}{2}\right)\right] \mathrm{d} t$,

may be either positive or negative.

In general, the same is true for the source term which may be either negative or positive. However, in the present setup, where the flow decelerates towards the stagnation point at the centre, 


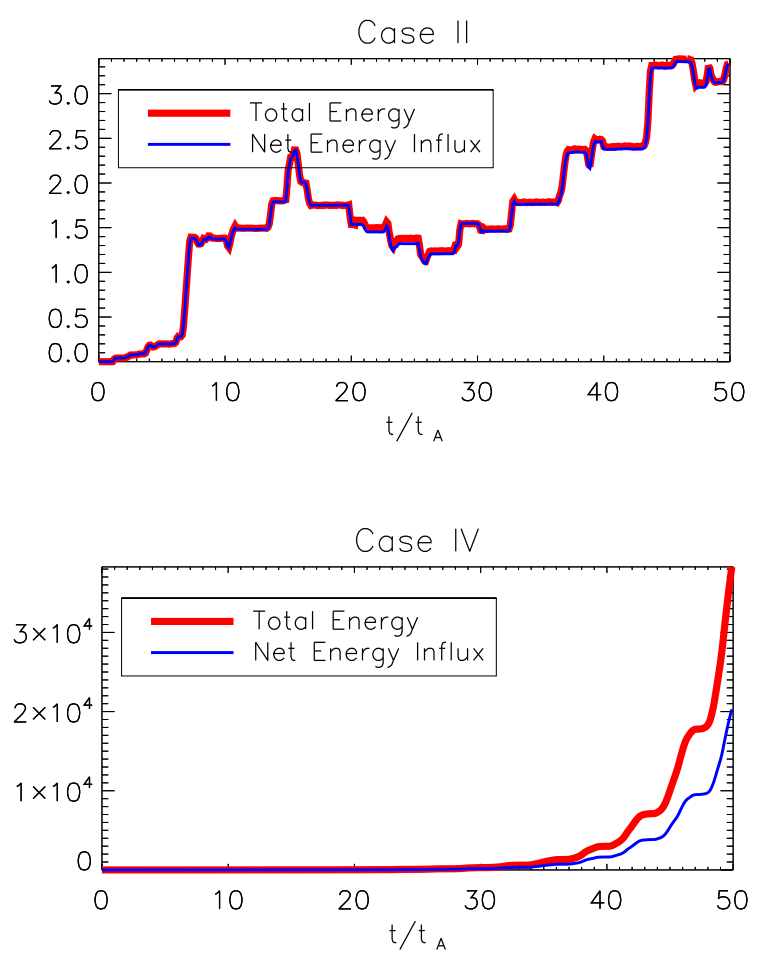

Fig. 9. Temporal evolution of total energy, $E_{\mathrm{T}}$, of the perturbations (thick red curve) and the time-integrated net influx of energy, $I_{\mathrm{E}}$, (thin blue curve) for Cases I-IV.

the right-hand side of Eq. (21) is always positive and it therefore acts as a source of energy due to the coupling between the flow and the twists along the thread. Energy extracted from the flow is transferred to the Alfvénic perturbations. One would expect a corresponding decrease in the longitudinal component of energy due to the same coupling process. However, the back reaction on the flow is ignored as the perturbations are small and the linear regime is considered.

Figure 9 displays the total energy, $E_{\mathrm{T}}$, and the net influx, $I_{\mathrm{E}}$, for Cases I-IV considered above. For Cases I and II, the two curves coincide as there is no source term due to the absence of flow. Any variation in energy is due to an influx through the two footpoints. The source of the incoming energy is the photospheric driver. Case II, where the driver is random, shows only moderate variation in energy after the perturbations set in at the start of the experiment.

The amplitude growth in Case I is caused by energy influx. Therefore, resonant amplification in a periodically driven system is a consequence of energy injection at the boundaries. In the solar physics context, this type of resonant amplification has been studied in relation to the generation of spicules (Sterling \& Hollweg 1984; Matsumoto \& Shibata 2010).

The lower panels in Fig. 9 for Cases III and IV show significantly stronger amplification compared with Case I. In both Cases, the growth is exponential. By the end of the experiments the total energy, $E_{\mathrm{T}}$, increases by 6 orders of magnitude in Case III and by 4-5 orders of magnitude in Case IV.

When the flow is added to the model, an additional term, $U W_{\mathrm{T}}$, appears on the right-hand side of the expression (8) for the energy flux. This term is always positive at the left footpoint and negative at the right footpoint. The influx, $I_{\mathrm{E}}$, is therefore less likely to become negative. The inflow leads to higher influx of energy once the perturbations set in.

The curves for $E_{\mathrm{T}}$ and $I_{\mathrm{E}}$ no longer coincide in the lower panels of Fig. 9, where the total energy is always higher than the net energy influx. The difference between the two curves is because of an additional contribution from the source term on the right-hand side of Eq. (21). This source term represents the coupling between the inflow and the torsional perturbations and it acts along the entire length of the thread.

The flow velocity, $U$, plays an important role in the amplification of perturbations. The perturbations will grow more rapidly if $U$ increases at the boundaries. This will be due to enhanced influx, $I_{\mathrm{E}}$, and due to steeper flow gradient which figures in the coupling term on the right-hand side of Eq. (21).

The temporal and spatial profile of the flow along the thread is likely to change when variations in magnetic field strength and density are taken into account. Both are expected to increase in a photosphere and chromosphere of finite thickness. The outcome, whether the perturbations vanish or amplify, will depend on the amount of influx and on the sign of the source term in Eq. (21).

\section{Conclusions}

A wave based mechanism for the generation of magnetic twists of a prominence thread is presented. The mechanism acts when the thread is permeated by a converging flow of plasma, for example, during prominence formation.

The swirls and tornadoes described in the Introduction are usually detected in intensity time series (Li et al. 2012; Wedemeyer-Böhm et al. 2012). The back reaction of the amplified twists on the mass flow will affect density. Forward modelling in the non-linear regime is required to investigate the evolution of density and the associated intensities.

A symmetric driver at the photosphere may lead to a quasistanding wave with growing amplitude, where the wave period increases in time due to decreasing Alfvén speed. However, it is important to note that the amplification of twists does not require a periodic driver at the footpoints as long as a converging flow 
towards the thread centre is present. The process of amplification is therefore different from a resonance.

Amplification may occur even when the total magnetic flux along the thread remains equal to zero. An influx of energy and the flow-twist coupling along the thread equally contribute to the amplification. Energy extracted from the converging flow is transferred into the perturbations.

The process of amplification will cease when the inflow is switched off. Otherwise, the process will continue. The twisting rate via the proposed mechanism depends on the flow speeds. High inflow speeds, such as those near active regions, may lead to strong twisting of the field lines and eventual eruption of the structure. The role of the amplified twists in the formation and eruption of prominences should be analysed using a multidimensional non-linear model.

Inclusion of a photosphere/chromosphere environment in the model will affect the flow profile in two different ways: a stronger magnetic field in the lower atmosphere is likely to increase the flow speed and its gradient, whereas a higher density is likely to have the opposite effect. The overall effect of a dense photosphere/chromosphere of finite depth is therefore not clear and requires a separate study.

The origin of the observed global kink-type oscillations of prominence threads remains unclear. It would be interesting to investigate the coupling of the plasma inflow to kink-type perturbations as a possible driver of these oscillations.

Acknowledgements. RS acknowledges the support from grant AYA201785465-P (MINECO/AEI/FEDER, UE) and from the Ministerio de Economía, Industria y Competitividad and the Conselleria d'Innovació, Recerca i Turisme del Govern Balear (Pla de ciència, tecnologia, innovació i emprenedoria 2013-2017) for the Ramón y Cajal grant RYC-2014-14970.

\section{References}

Arregui, I., Oliver, R., \& Ballester, J. L. 2018, Liv. Rev. Sol. Phys., 15, 154 Ballester, J. L., Carbonell, M., Soler, R., \& Terradas, J. 2018, A\&A, 609, A6
Carlsson, M., \& Stein, R. F. 1997, ApJ, 481, 500

Engvold, O. 2015, in Solar Prominences, eds. J. C. Vial, \& O. Engvold, Astrophys. Space Sci. Lib., 415, 31

Erdélyi, R., Al-Ghafri, K. S., \& Morton, R. J. 2011, Sol. Phys., 272, 73

Field, G. B. 1965, ApJ, 142, 531

Gibson, S. E. 2018, Liv. Rev. Sol. Phys., 15, 7

Hamdi, S., Schiesser, W. E., \& Griffiths, G. W. 2007, Scholarpedia, 2, 2859

Hillier, A., Morton, R. J., \& Erdélyi, R. 2013, ApJ, 779, L16

Joarder, P. S., \& Roberts, B. 1992a, A\&A, 256, 264

Joarder, P. S., \& Roberts, B. 1992b, A\&A, 261, 625

Karpen, J. T. 2015, in Solar Prominences, eds. J. C. Vial, \& O. Engvold, Astrophys. Space Sci. Lib., 415, 237

Lemen, J. R., Title, A. M., \& Akin, D. J. 2012, Sol. Phys., 275, L17

Luna, M., Karpen, J. T., \& DeVore, C. R. 2012, ApJ, 746, 30

Li, X., Morgan, H., Leonard, D., \& Jeska, L. 2012, ApJ, 752, L22

Lin, Y. 2011, Space Sci. Rev., 158, 237

Martin, S. F. 2015, in Solar Prominences, eds. J. C. Vial, \& O. Engvold, Astrophys. Space Sci. Lib., 415, 205

Matsumoto, T., \& Shibata, K. 2010, ApJ, 710, 1857

Parenti, S. 2014, Liv. Rev. Sol. Phys., 11, 1

Pike, C. D., \& Mason, H. E. 1998, Sol. Phys., 182, 333

Priest, E. R., Hood, A. W., \& Anzer, U. 1989, A\&A, 344, 1010

Rust, D. M., \& Kumar, A. 1994, Sol. Phys., 155, 69

Scharmer, G. B., Bjelksjo, K., Korhonen, T. K., Lindberg, B., \& Petterson, B. 2003, in Innovative Telescopes and Instrumentation for Solar Astrophysics, eds. S. L. Keil, \& S. V. Avakyan, Proc. SPIE, 4853, 341

Soler, R., Oliver, R., \& Ballester, J. L. 2008, ApJ, 684, 725

Soler, R., Arregui, I., Oliver, R., \& Ballester, J. L. 2010, ApJ, 722, 1778

Soler, R., Goossens, M., \& Ballester, J. L. 2015, A\&A, 575, A123

Sterling, A. C., \& Hollweg, J. V. 1984, ApJ, 285, 843

Taroyan, Y. 2015, A\&A, 575, A104

Taroyan, Y., \& Williams, T. 2016, ApJ, 829, 107

Terradas, J., Arregui, I., Oliver, R., \& Ballester, J. L. 2008, ApJ, 678, L153

Terradas, J., Soler, R., Luna, M., Oliver, R., \& Ballester, J. L. 2015, ApJ, 799, 94

Terradas, J., Soler, R., Luna, M., et al. 2016, ApJ, 820, 125

van Ballegooijen, A. A., \& Martens, P. C. H. 1989, ApJ, 343, 971

Vernazza, J. E., Avrett, E. H., \& Loeser, R. 1981, ApJS, 45, 635

Wedemeyer, S., Scullion, E., Rouppe van der Voort, L., Boskjak, A., \& Antolin, P. 2013, ApJ, 774, 123

Wedemeyer-Böhm, S., Scullion, E., Steiner, O., et al. 2012, Nature, 486, 505

Williams, T., \& Taroyan, Y. 2018, ApJ, 852, 77

Williams, T., Taroyan, Y., \& Fedun, V. 2016, ApJ, 817, 92 